九州大学学術情報リポジトリ

Kyushu University Institutional Repository

\title{
Variable selection for varying coefficient models with the sparse regularization
}

Matsui, Hidetoshi

Faculty of Mathematics, Kyushu University

Misumi, Toshihiro

Astellas Pharma Inc. | Graduate School of Science and Engineering, Chuo University

http://hdl. handle. net/2324/26215

出版情報: Computational Statistics. 29，2013-03-28. Springer Berlin Heidelberg バージョン：

権利関係 : 


\section{Preprint Series}

Kyushu University

The Global COE Program

Math-for-Industry Education \& Research Hub

\section{Variable selection for varying coefficient models with the sparse regularization}

\section{Hidetoshi Matsui}

MI 2013-8

( Received March 28, 2013 )

Faculty of Mathematics

Kyushu University

Fukuoka, JAPAN 


\title{
Variable selection for varying coefficient models with the sparse regularization
}

\author{
Hidetoshi Matsui ${ }^{\mathrm{a}}$ Toshihiro Misumi ${ }^{\mathrm{b}, \mathrm{c}}$ \\ ${ }^{a}$ Faculty of Mathematics, Kyushu University, \\ 744 Motooka, Nishi-ku, Fukuoka 819-0395, Japan, \\ b Astellas Pharma Inc., \\ 3-17-1, Hasune, Itabashi-ku, Tokyo 174-8612, Japan, \\ ${ }^{\mathrm{c}}$ Graduate School of Science and Engineering, Chuo University, \\ 1-13-27, Kasuga, Bunkyo-ku, Tokyo 112-8551, Japan. \\ hmatsui@math.kyushu-u.ac.jp (H. Matsui)
}

\begin{abstract}
Varying-coefficient models are useful tools for analyzing longitudinal data. They can effectively describe a relationship between predictors and responses repeatedly measured. We consider the problem of selecting variables in the varying-coefficient models via the adaptive elastic net regularization. Coefficients given as functions are expressed by basis expansions, and then parameters involved in the model are estimated by the penalized likelihood method using the coordinate descent algorithm derived for solving the problem of sparse regularization. We examine the effectiveness of our modeling procedure through Monte Carlo simulations and real data analysis.
\end{abstract}

Key Words and Phrases: Basis expansion, Elastic net, Group lasso, Varyingcoefficient model.

\section{Introduction}

Longitudinal data analysis has been used in various fields such as bioscience, ergonomics and meteorology. In longitudinal studies the data are measured repeatedly over time for individual and they have possibly different time points, which makes it difficult to directly apply the traditional multivariate analysis. Enormous works have contributed to the development of longitudinal data analysis during the past few decades (see, e.g. Diggle, 2002). In this article we focus on the problem of selecting variables which seem to have a relation with a response which is repeatedly measured.

Several methods have been proposed for the analysis of longitudinal data. Laird and Ware (1982) introduced random effects models and their estimation procedures and Zeger and Diggle (1994) applied semiparametric models as the regression models for longitudinal data. Ramsay and Silverman (2005) considered treating longitudinal data as smooth functions, and then established the Functional Data Analysis (FDA). On the other hand, Hoover et al. (1998) generalized the semiparametric model and applied the time varying-coefficient model. It is a special case of the varying-coefficient models (Hastie and Tibshirani, 1993) and obtained for analyzing longitudinal data. They approached 
the modeling by use of kernel smoothing or smoothing splines with an $L_{2}$-type penalty. Huang et al. (2002) also used smoothing splines for time varying-coefficient models and suggested a non-iterative solution for coefficient estimates. The effectiveness of varying coefficient models are reported in several works (Fan and Zhang, 1999; Cai et al., 2000; Eubank et al., 2004).

More recently, a class of sparse regularization has come to be used for the varyingcoefficient modeling. Sparse regularization including the lasso (Tibshirani, 1996), SCAD (Fan and Li, 2001) and the elastic net (Zou and Hastie, 2005) simultaneously shrinks parameters and selects variables which seem to be relevant to a response by estimating coefficients of rest of the variables to be exactly zeros, and therefore it is one of the most attractive methods in recent years. More details of sparse regularization can be seen in Hastie et al. (2009). We can apply the sparse regularization to the varying-coefficient models by expressing coefficient functions by basis expansions and then imposing $L_{1^{-}}$ type penalties to coefficient vectors. Since each variable has multiple parameters in this case, Wang et al. (2007b) treated this problem as the grouped variable selection and then applied the idea of the group lasso by Yuan and Lin (2006). They also selected the transcriptional factors involved in gene regulation during a biological process. Wei et al. (2011) applied the adaptive lasso penalty (Zou, 2006) and then estimated the model by the coordinate descent algorithm (Friedman et al., 2007) which is derived for the sparse regularization problem. Many other works concerning the varying-coefficient modeling with the sparse regularization have been reported (Wang et al., 2008; Noh and Park, 2010; Xue and Qu, 2012; Wang et al., 2013).

In this paper we propose a method for estimating and selecting models simultaneously by the varying-coefficient modeling along with the elastic net regularization by extending the method by Wei et al. (2011). Especially we use an adaptive elastic net penalty proposed by Zou and Zhang (2009). They combined the idea of the elastic net and the adaptive lasso in the framework of the general linear model. The adaptive elastic net regularization can select variables even if the number of variables is much larger than the sample size and can take into account highly correlated predictors. Furthermore its estimates has an preferable property called the "oracle property." By applying the elastic net regularization to the varying-coefficient model we can prevent its estimation from giving unstable or degenerate results owing to both $L_{1}$ and $L_{2}$ terms of the penalty. Coefficient functions of varying-coefficient models are expressed by basis expansions, and then parameters are estimated by the penalized maximum likelihood method with the help of the coordinate descent algorithm. In order to select tuning parameters involved in the adaptive elastic net penalty we use a Bayesian model selection criterion derived for evaluating the varying-coefficient model. The proposed modeling strategy is applied to Monte Carlo simulations and clinical investigation data to investigate the effectiveness of our method. 
This paper is organized as follows. In Section 2 we introduce a varying-coefficient model which describe the relationship between multiple predictors and a response repeatedly measured. Section 3 provides a method for estimating the varying-coefficient model with the adaptive elastic net regularization and selecting tuning parameters. Monte Carlo simulations are conducted in Section 4 in order to evaluate the effectiveness of the proposed modeling strategy and real data analysis are described in Section 5. Finally concluding remarks are given in Section 6.

\section{Varying-coefficient models}

Suppose we have $p$ sets of predictors $X_{k}(k=1, \ldots, p)$ and a response $Y$, each of them are repeatedly observed at possibly different time points. Denote $i$-th of $n$ individuals at $j$-th of $n_{i}$ time points of $X_{k}$ and $Y$ as $x_{i j k}$ and $y_{i j}$ respectively. The varying-coefficient model (Hoover et al., 1998) that represents the relationship between $X_{k}$ s and $Y$ is given by

$$
y_{i j}=\beta_{0}\left(t_{i j}\right)+x_{i j 1} \beta_{1}\left(t_{i j}\right)+\cdots+x_{i j p} \beta_{p}\left(t_{i j}\right)+\varepsilon_{i j},
$$

where $\beta_{0}(\cdot)$ is an intercept and $\beta_{1}(\cdot), \ldots, \beta_{p}(\cdot)$ are coefficients, both of which are given as functions to be estimated. Moreover, $\varepsilon_{i j}$ are random noises whose vector $\varepsilon_{i}=\left(\varepsilon_{i 1}, \ldots, \varepsilon_{i n_{i}}\right)^{\prime}$ are normally distributed with mean vector $\mathbf{0}$ and variance covariance matrix $\sigma^{2} S_{i}$ with unknown scalar $\sigma^{2}$ and known $n_{i} \times n_{i}$ symmetric matrix $S_{i}$.

We assume that coefficient functions $\beta_{k}(\cdot)(k=0, \ldots, p)$ are expressed by basis expansions as follows;

$$
\beta_{k}\left(t_{i j}\right)=\sum_{m=1}^{M_{k}} \gamma_{k m} \phi_{m}^{(k)}\left(t_{i j}\right)=\gamma_{k}^{\prime} \phi^{(k)}\left(t_{i j}\right)
$$

where $\gamma_{k}=\left(\gamma_{k 1}, \ldots, \gamma_{k M_{k}}\right)^{\prime}$ are coefficient vectors and $\phi^{(k)}\left(t_{i j}\right)=\left(\phi_{1}^{(k)}\left(t_{i j}\right), \ldots, \phi_{M_{k}}^{(k)}\left(t_{i j}\right)\right)^{\prime}$ are basis functions. There are several basis functions available for $\phi^{(k)}(t)$ such as wavelets or radial basis functions. Here we apply cubic $B$-spline bases, whose details are referred to de Boor (2001); Imoto and Konishi (2003). Using this assumption and denoting $\boldsymbol{y}_{i}=\left(y_{i 1}, \ldots, y_{i n_{i}}\right)^{\prime}, D_{i k}=\operatorname{diag}\left(x_{i 1 k}, \ldots, x_{i n_{i} k}\right)(k=1, \ldots, p), D_{i 0}=I_{n_{i}}$ and $\Phi_{i k}=\left(\phi^{(k)}\left(t_{i 1}\right), \ldots, \phi^{(k)}\left(t_{i n_{i}}\right)\right)^{\prime}$, the varying-coefficient model (1) can be rewritten as

$$
\boldsymbol{y}_{i}=\sum_{k=0}^{p} D_{i k} \Phi_{i k} \gamma_{k}+\varepsilon_{i}, \quad \boldsymbol{\varepsilon}_{i} \sim N_{n_{i}}\left(\mathbf{0}, \sigma^{2} S_{i}\right)
$$

Then we have a probability density function

$f\left(\boldsymbol{y}_{i} \mid \boldsymbol{\theta}\right)=\frac{1}{\left(2 \pi \sigma^{2}\right)^{n_{i} / 2}\left|S_{i}\right|^{1 / 2}} \exp \left\{-\frac{1}{2 \sigma^{2}}\left(\boldsymbol{y}_{i}-\sum_{k=0}^{p} D_{i k} \Phi_{i k} \boldsymbol{\gamma}_{k}\right)^{\prime} S_{i}^{-1}\left(\boldsymbol{y}_{i}-\sum_{k=0}^{p} D_{i k} \Phi_{i k} \boldsymbol{\gamma}_{k}\right)\right\}$ 
where we denote a vector of unknown parameters by $\boldsymbol{\theta}=\left\{\gamma_{0}, \ldots, \boldsymbol{\gamma}_{p}, \sigma^{2}\right\}$. After obtaining an estimator of $\boldsymbol{\theta}$, denoted by $\hat{\boldsymbol{\theta}}$, by the method described in the next section, we have a statistical model $f\left(\boldsymbol{y}_{i} \mid \hat{\boldsymbol{\theta}}\right)$.

\section{Estimation and evaluation via the sparse regular- ization}

Unknown parameters; coefficient vectors $\gamma_{k}$ and variance parameter $\sigma^{2}$, involved in the varying coefficient model are estimated by the maximum penalized likelihood method which maximizes a penalized log-likelihood function. The penalized log-likelihood function is given in the form of

$$
l_{\lambda}(\boldsymbol{\theta})=\sum_{i=1}^{n} \log f\left(\boldsymbol{y}_{i} \mid \boldsymbol{\theta}\right)-n \lambda \sum_{k=1}^{p} P_{\alpha}\left(\left\|\boldsymbol{\gamma}_{k}\right\|\right),
$$

where $P_{\alpha}(\cdot)$ is a penalty function, $\|\cdot\|$ is a standard $L_{2}$ norm and $\lambda>0$ is a regularization parameter which controls the degree of the penalty. We impose the penalty composed by a sum of penalty functions of $L_{2}$ norms of the coefficient vectors $\gamma_{k}$ instead of its components separately. Then we can shrink all elements of the vector $\gamma_{k}$ towards exactly zeros when the corresponding predictor seems to be less relevant to the response. It is exactly the idea of the group lasso by Yuan and Lin (2006). For the penalty function $P_{\alpha}$ we use an adaptive elastic net penalty (Zou and Zhang, 2009) given by

$$
P_{\alpha}(\theta)=\frac{1}{2}(1-\alpha) \theta^{2}+\alpha \hat{w}_{k}|\theta|
$$

for all $\theta \in \mathbb{R}$, where $\alpha \in[0,1]$ tunes the type of the penalty between the ridge $(\alpha=0)$ and the lasso $(\alpha=1)$. Furthermore, $\hat{w}_{k}>0$ is an adaptive weight which is given in the form of $\hat{w}_{k}=\left(\sqrt{M_{k}}\left\|\gamma_{k}\right\|\right)^{-\beta}\left(\left\|\gamma_{k}\right\| \neq 0\right),=\infty\left(\left\|\gamma_{k}\right\|=0\right)$ with a positive constant $\beta$, where we use $\beta=1$ in this paper.

We have difficulty in analytical expressions for the estimates of coefficients of regression models via the $L_{1}$-type regularization. Wang et al. (2007b) used a local quadratic approximation (Fan and Li, 2001) for the varying-coefficient modeling via the group SCAD regularization, and Wei et al. (2011) applied a coordinate descent procedure (Friedman et al., 2007) to the group adaptive lasso regularization. The coordinate descent method has similar algorithm to the backfitting algorithm, used in the varying coefficient modeling (Hastie and Tibshirani, 1993), in that it updates coefficients of each variable in turn. Using this method, we obtain updated values of $k$-th coefficients $(k=1, \ldots, p)$ as

$$
\hat{\boldsymbol{\gamma}}_{k}=\left(\sum_{i=1}^{n} \Phi_{i k}^{\prime} D_{i k} S_{i}^{-1} D_{i k} \Phi_{i k}+n(1-\alpha) \lambda \hat{\sigma}^{2} I\right)^{-1}\left(\left\|\boldsymbol{\zeta}_{k}\right\|-n \alpha \lambda \hat{w}_{k} \hat{\sigma}^{2}\right)_{+} \frac{\boldsymbol{\zeta}_{k}}{\left\|\boldsymbol{\zeta}_{k}\right\|}
$$


where

$$
\boldsymbol{\zeta}_{k}=\sum_{i=1}^{n} \Phi_{i k}^{\prime} D_{i k} S_{i}^{-1}\left(\boldsymbol{y}_{i}-\sum_{l \neq k}^{p} D_{i l} \Phi_{i l} \hat{\gamma}_{l}\right)
$$

and $z_{+}=\max \{z, 0\}$ for $z \in \mathbb{R}$. Both of two terms of the adaptive elastic net penalty (3) directly have effects to the estimates (4) of the varying-coefficient model. The $L_{1}$ term of the penalty shrinks some of coefficients $\gamma_{k}$ towards zero vectors, while the $L_{2}$ term prevents the degeneracy of the inverse, especially when there are few time points for individual and a large number of basis functions. Although the first term in the inverse matrix of (4) does not always degenerate even if $n<p$ unlike the ordinal linear model, the $L_{2}$ term of the elastic net penalty provides stable estimates. Note that when $k=0$ there is no regularization and therefore $\hat{\gamma}_{0}$ has the form of (4) with a generalized inverse and $\lambda=0$. The variance parameter is then estimated in the following form:

$$
\hat{\sigma}^{2}=\frac{1}{\sum_{i} n_{i}} \sum_{i=1}^{n}\left(\boldsymbol{y}_{i}-\sum_{k=0}^{p} D_{i k} \Phi_{i k} \hat{\gamma}_{k}\right)^{\prime} S_{i}^{-1}\left(\boldsymbol{y}_{i}-\sum_{k=0}^{p} D_{i k} \Phi_{i k} \hat{\gamma}_{k}\right)
$$

Since $\hat{\gamma}_{k}$ and $\hat{\sigma}^{2}$ depend on each other, they are updated until a convergence condition is achieved. Consequently, the algorithm is given as follows:

1. Initialize parameters $\gamma_{0}, \gamma_{1}, \ldots, \gamma_{p}$ and $\sigma^{2}$ by 1 for all elements.

2. For $k=0,1 \ldots, p$, update $\gamma_{k}$ by (4) in turn.

3. Update $\sigma^{2}$ by (5).

4. Iterate 2 and 3 until convergence.

Thus the statistical model is obtained by substituting the estimators $\hat{\boldsymbol{\theta}}=\left\{\hat{\boldsymbol{\gamma}}_{0}, \ldots, \hat{\gamma}_{p}, \hat{\sigma}^{2}\right\}$ given above into the probability density function (2), that is,

$f\left(\boldsymbol{y}_{i} \mid \hat{\boldsymbol{\theta}}\right)=\frac{1}{\left(2 \pi \hat{\sigma}^{2}\right)^{n_{i} / 2}\left|S_{i}\right|^{1 / 2}} \exp \left\{-\frac{1}{2 \hat{\sigma}^{2}}\left(\boldsymbol{y}_{i}-\sum_{k=0}^{p} D_{i k} \Phi_{i k} \hat{\gamma}_{k}\right)^{\prime} S_{i}^{-1}\left(\boldsymbol{y}_{i}-\sum_{k=0}^{p} D_{i k} \Phi_{i k} \hat{\gamma}_{k}\right)\right\}$.

The statistical model estimated by the adaptive elastic net regularization depends on the regularization parameter, the tuning parameter and the number of basis functions, therefore we need to decide these values objectively. The decision problem is regarded as a model selection or evaluation problem. Although a cross validation is widely used for the model selection problem, it often selects unstable estimates and has high computational burden. Wang et al. (2007a) showed that GCV does not select the true model consistently and that BIC consistently select the true model for the SCAD regularization. We apply 
the BIC for selecting the regularization parameter $\lambda$, tuning parameter $\alpha$ and the number of basis functions $M_{k}$. Model selection criterion BIC has the form of

$$
\begin{aligned}
\mathrm{BIC} & =-2 \sum_{i=1}^{n} \log f(Y \mid \hat{\boldsymbol{\theta}})+e d f \log n \\
& =-\log \left(2 \pi \hat{\sigma}^{2}\right) \sum_{i=1}^{n} n_{i}-\sum_{i=1}^{n} \log \left|S_{i}\right|-\sum_{i=1}^{n} n_{i}+e d f \log n
\end{aligned}
$$

where edf is an effective degrees of freedom for the varying-coefficient model. Matsui et al. (2013) derived an effective degrees of freedom for the varying coefficient models estimated by the regularization method with the $L_{2}$ penalty. Applying this result, the

effective degrees of freedom for the varying coefficient models estimated by the adaptive elastic net regularization is given by

$$
\begin{array}{r}
e d f=\sum_{i=1}^{n} \sum_{k=1}^{p} \operatorname{tr}\left\{D_{i k} \Phi_{i k}\left(\sum_{i=1}^{n} \Phi_{i k}^{\prime} D_{i k} S_{i}^{-1} D_{i k} \Phi_{i k}+n(1-\alpha) \lambda \hat{\sigma}^{2} I_{M_{k}}\right)^{-1}\right. \\
\left.\left(\left\|\boldsymbol{\zeta}_{k}\right\|-n \alpha \lambda \hat{w}_{k} \hat{\sigma}^{2}\right)_{+} \frac{1}{\left\|\boldsymbol{\zeta}_{k}\right\|} \Phi_{i k}^{\prime} D_{i k} S_{i}^{-1}\right\} .
\end{array}
$$

\section{Simulation study}

Monte Carlo simulations are conducted in order to examine the effectiveness of our modeling procedure. We generated a repeated measurement data set $\left\{\left(\boldsymbol{x}_{i j}, y_{i j}\right) ; i=\right.$ $\left.1, \ldots, n, j=1, \ldots, n_{i}\right\}$, where $\boldsymbol{x}_{i j}=\left(x_{i j 0}, x_{i j 1}, \ldots, x_{i j p}\right)^{\prime}$ with $x_{i j 0}=1$, from a true model

$$
\begin{aligned}
& y_{i j}=f\left(\boldsymbol{x}_{i j}\right)+\varepsilon_{i j}, \quad f\left(\boldsymbol{x}_{i j}\right)=\sum_{k=0}^{p} x_{i j k} \beta_{k}\left(t_{i j}\right), \\
& \varepsilon_{i j} \sim N\left(0, \sigma^{2}\right), \quad \sigma=s\left\{\max _{i, j} f\left(\boldsymbol{x}_{i j}\right)-\min _{i, j} f\left(\boldsymbol{x}_{i j}\right)\right\},
\end{aligned}
$$

where we set the number of predictors to be $p=50$, which only first 30 predictors are relevant to the response. We investigated whether the proposed model appropriately select predictors which is relevant to the response for various values of sample sizes $n$ and variance parameters $s$.

First, time points $t_{i j}$ were generated from an uniform distribution $U(-0.5,0.5)$ and their numbers $n_{i}$ were uniformly generated as integer values between 4 and 15 for each subject $i$. Then predictors $X_{k}=X_{k}(t)$ were generated by following random numbers:

$$
\begin{array}{rlrl}
X_{1}(t) & \sim U(t / 10,2+t / 10), & X_{7}(t) & \sim N(3 \exp (t / 30), 1), \\
X_{13}(t) & \sim N(t, 1), & X_{19}(t) & \sim N(\sin (2 \pi t)+2,1), \\
X_{25}(t) & \sim N(\cos (2 \pi t)+2,1) &
\end{array}
$$


for $k=1,7,13,19,25$ respectively. They are independent of any other variables. Other variables for $k \leq 30$, which are dependent on above variables, are generated from $N((1+$ $\left.\left.x_{i k^{\prime}}(t)\right) /\left(2+x_{i k^{\prime}}(t)\right), 1\right)$ where $k^{\prime}=6\lfloor(k-1) / 6\rfloor+1$ with a floor function $\lfloor\cdot\rfloor$ for each $i$. Remaining variables $X_{k}(t)$ for $k=31, \ldots, 50$, independent of each other, are generated from a multivariate normal distribution with mean vector $\mathbf{0}$ and variance covariance matrix $\operatorname{cov}\left(X_{k}(t), X_{k}(s)\right)=4 \exp (-|t-s| / 5)$. Next, coefficient functions are set to be

$$
\begin{aligned}
\beta_{0}(t) & =\exp \left(t^{3}\right), & & \beta_{1}(t)=2+3 \sin (\pi t / 60), \\
\beta_{6}(t) & =2+3 \cos (\pi t / 60), & & \beta_{11}(t)=2-3 \sin (\pi(t-25) / 15), \\
\beta_{16}(t) & =2-3 \cos (\pi(t-25) / 15), & & \beta_{21}(t)=6-0.2 t^{2}, \\
\beta_{26}(t) & =-4+(20-t)^{3} / 2000 . & &
\end{aligned}
$$

Other coefficients for $k \leq 30$ are obtained from

$$
\beta_{k}(t)=\beta_{k^{\prime \prime}}(t)+\boldsymbol{\eta}^{\prime} \boldsymbol{\psi}(t)
$$

where $k^{\prime \prime}=5\lfloor(k-1) / 5\rfloor+1$ and $\boldsymbol{\psi}(t)=\left(\psi_{1}(t), \ldots, \psi_{5}(t)\right)^{\prime}$ is a vector of cubic $B$-spline basis functions and $\boldsymbol{\eta}=\left(\eta_{1}, \ldots, \eta_{5}\right)^{\prime}$ is a random variable which follows multivariate normal distribution with mean vector $\mathbf{0}$ and variance covariance matrix $\operatorname{cov}\left(\eta_{i}, \eta_{j}\right)=$ $4 \exp (-|i-j| / 5)$. The remaining coefficients are set to be $\beta_{k}(t)=0$ for $k=31, \ldots, 50$, which suggest that the variables $X_{k}$ for $k=31, \ldots, 50$ are irrelevant to the response. We considered three patterns of sample size $n=15,25,50$ and two patterns of variance parameter $s=0.05,0.1$.

For the data set, we applied our varying-coefficient modeling procedure with cubic $B$-spline basis, here we assumed that the number of basis functions $M_{k}=6$ for all $k$. Regularization parameters in the penalized log-likelihood function were selected by BIC given in (6). In order to investigate the effectiveness of the proposed method, we compared results of our modeling procedure with those of the adaptive group lasso. We repeated this procedure for 100 times, and then obtained 100 mean squared errors MSE $=\sum_{i} \sum_{j}\left\{f\left(\boldsymbol{x}_{i j}\right)-\hat{y}_{i j}\right\}^{2} / \sum_{i} n_{i}$, where $\hat{y}_{i j}$ is a predictive value of $y_{i j}$. Furthermore we examined numbers of selected variables and proportions of variables correctly estimated by zero and incorrectly estimated by zero so as to investigate the variable selection performance.

Table 1 shows results of simulation studies. It contains averages and standard deviations of 100 MSEs and selected regularization parameters and averages of tuning parameters. This table also provides numbers of selected variables and proportions that were correctly and incorrectly estimated to be zeros. These results show that our method gives fewer and stable (or competitive) MSEs than the adaptive lasso. The number of selected variables of the adaptive elastic net are larger than that of the adaptive lasso. The proportions of variables correctly estimated to be zeros are fewer than the adaptive lasso, 
Table 1: Result of simulation studies. Notations "ave" and "sd" represent average and standard deviation respectively.

\begin{tabular}{ccccccc}
\hline \hline & \multicolumn{3}{c}{ Adaptive elastic net } & \multicolumn{3}{c}{ Adaptive lasso } \\
\hline$n$ & 15 & 25 & 50 & 15 & 25 & 50 \\
\hline $\mathrm{s}=0.05$ & & & & & & \\
ave $(\mathrm{MSE}) \times 10^{-2}$ & 0.91 & 2.43 & 2.60 & 1.66 & 3.49 & 3.37 \\
$\mathrm{sd}(\mathrm{MSE}) \times 10^{-2}$ & 0.76 & 1.27 & 1.01 & 1.60 & 1.55 & 1.26 \\
ave $(\lambda) \times 10^{2}$ & 4.21 & 4.17 & 2.20 & 5.96 & 6.17 & 2.85 \\
$\mathrm{sd}(\lambda) \times 10^{2}$ & 1.48 & 1.17 & 0.35 & 3.45 & 2.47 & 1.47 \\
ave $(\alpha)$ & 0.85 & 0.80 & 0.80 & - & - & - \\
select & 32.42 & 24.18 & 25.55 & 23.10 & 9.19 & 12.49 \\
correct & 35.85 & 67.15 & 67.95 & 50.55 & 92.70 & 89.90 \\
incorrect & 38.03 & 44.63 & 39.53 & 59.30 & 77.57 & 68.43 \\
\hline $\mathrm{s}=0.1$ & & & & & & \\
ave $(\mathrm{MSE}) \times 10^{-2}$ & 1.59 & 2.61 & 3.04 & 2.37 & 3.37 & 3.98 \\
$\mathrm{sd}(\mathrm{MSE}) \times 10^{-2}$ & 1.38 & 1.39 & 1.33 & 1.78 & 1.56 & 1.38 \\
ave $(\lambda) \times 10^{2}$ & 3.97 & 4.04 & 2.25 & 5.70 & 5.26 & 3.50 \\
sd $(\lambda) \times 10^{2}$ & 1.11 & 1.07 & 0.49 & 3.33 & 1.89 & 0.20 \\
ave $(\alpha)$ & 0.85 & 0.80 & 0.80 & - & - & - \\
select & 31.68 & 23.90 & 24.59 & 22.00 & 10.24 & 10.57 \\
correct & 38.95 & 66.60 & 71.55 & 55.90 & 89.10 & 94.05 \\
incorrect & 38.43 & 45.93 & 40.33 & 59.40 & 76.47 & 72.07 \\
\hline
\end{tabular}

while those incorrectly estimated to be zeros are larger. It suggests that the adaptive elastic net tends to select necessary variables than the adaptive lasso.

\section{$5 \quad$ Real data example}

We applied the proposed modeling strategy to the analysis of the multicenter AIDS cohort study data, available on the $\mathrm{R}$ package timereg. The aim of the analysis is to investigate the relationship between properties of the human who are infected with the Human Immunodeficiency Virus (HIV) and the percentages of the CD4 cells in their blood. The data set contains cigarette smoking status, age at HIV infection, pre-HIV infection CD4 cell percent and the CD4 cell percentage of each subject, observed at distinct time points after HIV infection. Several researchers have applied the varying-coefficient modeling to the analysis of this data (Fan and Zhang, 2000; Huang et al., 2004; Wang et al., 2008). We applied the varying coefficient model and the group adaptive elastic net regularization in order to investigate which combination of variables is important.

We represent the relationship of variables described above by the time varying-coefficient 
Table 2: Selected numbers of each variable for 100 bootstrap samples. " $k$ " denotes the number of the variable. The upper side of the table indicates the result of original variables, while the lower is of artificially appended variables.

\begin{tabular}{ccccccccccc}
\hline \hline$k$ & 1 & 2 & 3 & 4 & & & & & & \\
\hline select & 0 & 0 & 6 & 100 & & & & & & \\
\hline \hline$k$ & 5 & 6 & 7 & 8 & 9 & 10 & 11 & 12 & 13 & 14 \\
\hline select & 0 & 0 & 0 & 22 & 22 & 4 & 0 & 0 & 6 & 4 \\
\hline
\end{tabular}

model written by

$$
y_{i}(t)=\sum_{k=0}^{p} x_{i k} \beta_{k}(t)+\varepsilon_{i}(t),
$$

where predictors $x_{i k}$ represent an overall intercept or baseline (BASE, $k=0$ ), observed time (TIME, $k=1$ ), age at HIV infection (AGE, $k=2$ ), cigarette smoking status represented by 0 or 1 (SMOKE, $k=3$ ), and pre-infection CD4 percent (PreCD4, $k=4$ ) of the $i$-th subject, respectively, response $y_{i}(t)$ is the CD4 percent of the $i$-th subject observed at differing time points, $\beta_{k}(t)$ are time varying coefficients and $\varepsilon_{i}(t)$ are the error functions. We assumed that the variance covariance matrix of the vector $\left(\varepsilon_{i}\left(t_{i 1}\right), \ldots, \varepsilon_{i}\left(t_{i n_{i}}\right)\right)^{\prime}$ were $\sigma^{2} I$. In addition to these original predictors, we appended 10 artificial variables to the predictor by the following method. First, we randomly selected a variable from $X_{k}, k=$ $2,3,4$ and then assigned a random sample of the selected variable without replacement to a new variable. We repeated this process for 10 times and then treated them as $X_{k}$ $(k=5, \ldots, 14)$.

Coefficient functions are supposed to be expressed by cubic $B$-spline basis expansions. Then the model was fitted by the maximum penalized likelihood method with group adaptive elastic net penalty, and it was evaluated by BIC. In order to avoid time consuming process, we assumed that the number of basis functions was the same among variables and the number of basis functions was set to be 6 . We generated 100 sets of bootstrap samples from the data, and then obtained 100 estimates of coefficient functions and numbers of selected variables.

Table 2 shows sums of numbers of selected variables except for BASE (intercept) for 100 repetition. We can find that PreCD4 was most selected and other original predictors were hardly selected. In addition, artificially appended variables were less selected. These results show that PreCD4 strongly has an influence on CD4 cell percentage, while other variables such as SMOKE and AGE have little relevance. Furthermore the proposed method can also appropriately exclude variables which are truly unnecessary.

Estimated coefficients are shown in Figure 1. Solid lines are mean coefficient functions for 100 bootstrap samples and dashed lines are pointwise $90 \%$ confidence intervals. Figure 

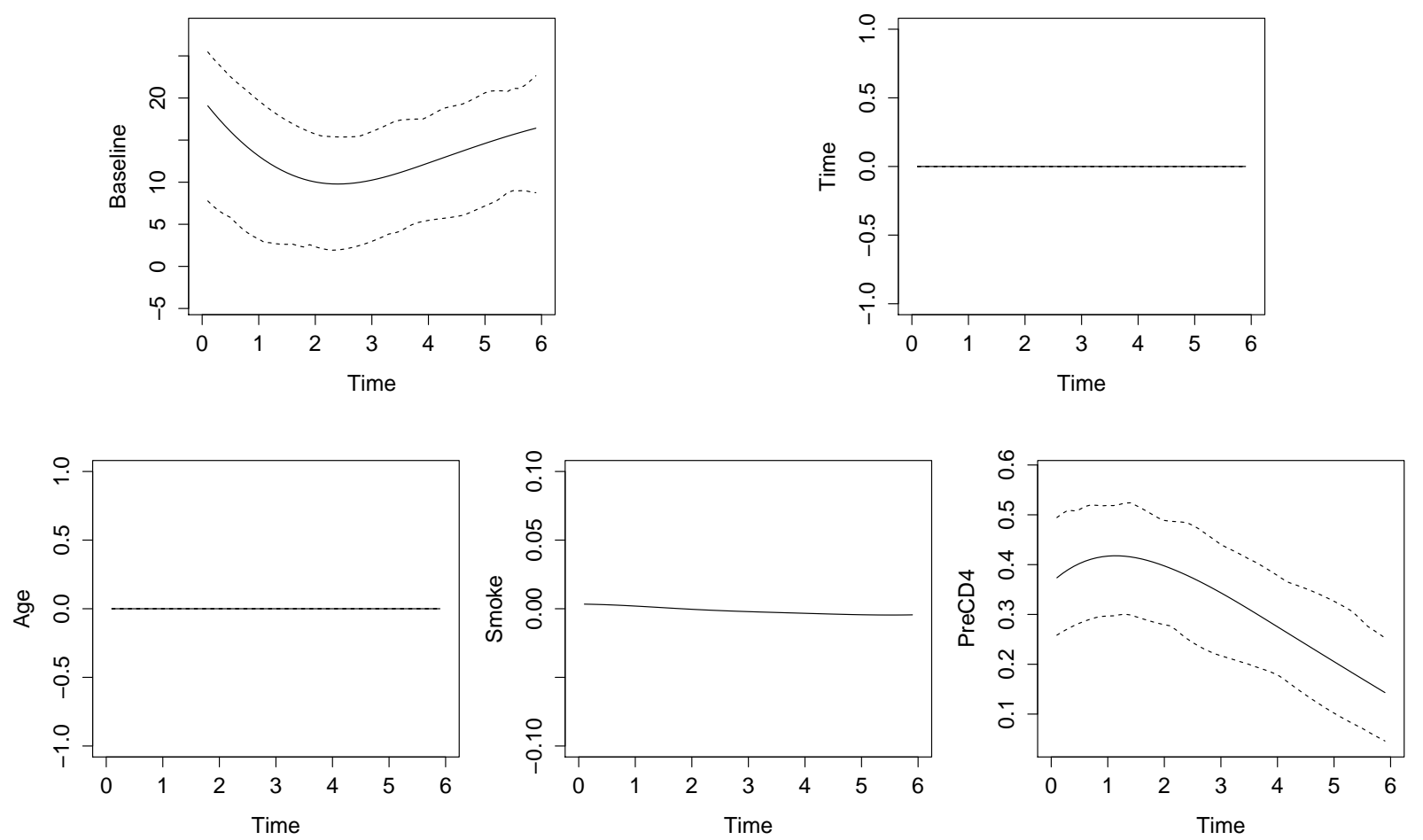

Figure 1: Estimated coefficient functions for original variables. Top left: BASE, top right: TIME, bottom left: AGE, bottom middle: SMOKE, bottom right: PreCD4.

1 suggests that the CD4 data have a trend that decreases with time in early time and then increases gradually, and that PreCD4 has a positive influence on CD4 cell percentage, but it gradually becomes weak with time. On the other hand, TIME, AGE and SMOKE seem to have almost no effect on the CD4 percentage all the time since most coefficients of them are estimated to be exactly zeros. It reveals that these three variables are irrelevant to the CD4 cell percentage. These results are quite similar to those of Wang et al. (2008).

\section{Concluding Remarks}

In this article, we have applied the sparse regularization to varying-coefficient models in order to select variables repeatedly measured at possibly different time points. We used an adaptive elastic net penalty in the penalized likelihood method, and then parameters involved in the model are estimated by the framework of the coordinate descent algorithm. In order to select regularization parameters involved in the penalized likelihood method, we derived an effective degrees of freedom for the varying coefficient model and then apply a BIC-type criterion. Simulation studies suggest that our modeling strategy worked well in the viewpoint of variable selection and prediction accuracy rather than the existing method. Furthermore we applied it to the analysis of real data and showed that some of information of the HIV patient seems to irrelevant to the CD4 cell percentage. 
More recently, Şentürk and Müller (2010) proposed a new type of varying-coefficient models which considers the relationship between a response and predictors with recent time points. Future work will focus on applying the sparse regularization to such models to investigate the time range that the predictors affect the response.

\section{References}

Cai, Z., Fan, J., and Li, R. (2000), "Efficient estimation and inferences for varyingcoefficient models," J. Amer. Statist. Assoc., 95, 888-902.

de Boor, C. (2001), A practical guide to splines, New York: Springer Verlag.

Diggle, P. (2002), Analysis of longitudinal data, Oxford: Oxford University Press.

Eubank, R., Huang, C., Maldonado, Y., Wang, N., Wang, S., and Buchanan, R. (2004), "Smoothing spline estimation in varying-coefficient models," J. Roy. Statist. Soc. Ser. B., 66, 653-667.

Fan, J. and Li, R. (2001), "Variable selection via nonconcave penalized likelihood and its oracle properties," J. Amer. Statist. Assoc., 96, 1348-1360.

Fan, J. and Zhang, J. (2000), "Two-step estimation of functional linear models with applications to longitudinal data," J. Roy. Statist. Soc. Ser. B., 62, 303-322.

Fan, J. and Zhang, W. (1999), "Statistical estimation in varying coefficient models," Ann. Statist., 27, 1491-1518.

Friedman, J., Hastie, T., Höfling, H., and Tibshirani, R. (2007), "Pathwise coordinate optimization," Ann. Appl. Statist., 1, 302-332.

Hastie, T. and Tibshirani, R. (1993), "Varying-coefficient models," J. Roy. Statist. Soc. Ser. B., 55, 757-796.

Hastie, T., Tibshirani, R., and Friedman, J. (2009), The elements of statistical learning 2nd ed., New York: Springer.

Hoover, D., Rice, J., Wu, C., and Yang, L. (1998), "Nonparametric smoothing estimates of time-varying coefficient models with longitudinal data," Biometrika, 85, 809-822.

Huang, J., Wu, C., and Zhou, L. (2002), "Varying-coefficient models and basis function approximations for the analysis of repeated measurements," Biometrika, 89, 111-128.

- (2004), "Polynomial spline estimation and inference for varying coefficient models with longitudinal data," Statist. Sinica, 14, 763-788. 
Imoto, S. and Konishi, S. (2003), "Selection of smoothing parameters in $B$-spline nonparametric regression models using information criteria," Ann. Inst. Statist. Math., 55, $671-687$.

Laird, N. and Ware, J. (1982), "Random-effects models for longitudinal data," Biometrics, 963-974.

Matsui, H., Misumi, T., and Kawano, S. (2013), "Model selection criteria for the varyingcoefficient modeling via regularized basis expansions," To appear in J. Statist. Comput. Simulation.

Noh, H. and Park, B. (2010), "Sparse varying coefficient models for longitudinal data," Statist. Sinica, 20, 1183-1202.

Ramsay, J. and Silverman, B. (2005), Functional data analysis, New York: Springer Verlag.

Şentürk, D. and Müller, H. (2010), "Functional varying coefficient models for longitudinal data," J. Amer. Statist. Assoc., 105, 1256-1264.

Tibshirani, R. (1996), "Regression shrinkage and selection via the lasso," J. Roy. Statist. Soc. Ser. B., 58, 267-288.

Wang, H., Li, R., and Tsai, C. (2007a), "Tuning parameter selectors for the smoothly clipped absolute deviation method," Biometrika, 94, 553-568.

Wang, H., Zou, G., and Wan, A. (2013), "Adaptive LASSO for varying-coefficient partially linear measurement error models," J. Statist. Plann. Inference, 143, 40-54.

Wang, L., Chen, G., and Li, H. (2007b), "Group SCAD regression analysis for microarray time course gene expression data," Bioinformatics, 23, 1486-1494.

Wang, L., Li, H., and Huang, J. (2008), "Variable selection in nonparametric varyingcoefficient models for analysis of repeated measurements," J. Amer. Statist. Assoc., 103, 1556-1569.

Wei, F., Huang, J., and Li, H. (2011), "Variable selection and estimation in highdimensional varying-coefficient models," Statist. Sinica, 21, 1515-1540.

Xue, L. and Qu, A. (2012), "Variable Selection in High-dimensional Varying-coefficient Models with Global Optimality," J. Mach. Learn. Res., 13, 1973-1998.

Yuan, M. and Lin, Y. (2006), "Model selection and estimation in regression with grouped variables," J. Roy. Statist. Soc. Ser. B., 68, 49-67. 
Zeger, S. and Diggle, P. (1994), "Semiparametric models for longitudinal data with application to CD4 cell numbers in HIV seroconverters," Biometrics, 50, 689-699.

Zou, H. (2006), "The adaptive lasso and its oracle properties," J. Amer. Statist. Assoc., $101,1418-1429$.

Zou, H. and Hastie, T. (2005), "Regularization and variable selection via the elastic net," J. Roy. Statist. Soc. Ser. B., 67, 301-320.

Zou, H. and Zhang, H. (2009), "On the adaptive elastic-net with a diverging number of parameters," Ann. Statist., 37, 1733. 


\section{List of MI Preprint Series, Kyushu University \\ The Global COE Program \\ Math-for-Industry Education \& Research Hub}

MI

MI2008-1 Takahiro ITO, Shuichi INOKUCHI \& Yoshihiro MIZOGUCHI

Abstract collision systems simulated by cellular automata

MI2008-2 Eiji ONODERA

The intial value problem for a third-order dispersive flow into compact almost Hermitian manifolds

MI2008-3 Hiroaki KIDO

On isosceles sets in the 4-dimensional Euclidean space

MI2008-4 Hirofumi NOTSU

Numerical computations of cavity flow problems by a pressure stabilized characteristiccurve finite element scheme

MI2008-5 Yoshiyasu OZEKI

Torsion points of abelian varieties with values in nfinite extensions over a p-adic field

MI2008-6 Yoshiyuki TOMIYAMA

Lifting Galois representations over arbitrary number fields

MI2008-7 Takehiro HIROTSU \& Setsuo TANIGUCHI

The random walk model revisited

MI2008-8 Silvia GANDY, Masaaki KANNO, Hirokazu ANAI \& Kazuhiro YOKOYAMA

Optimizing a particular real root of a polynomial by a special cylindrical algebraic decomposition

MI2008-9 Kazufumi KIMOTO, Sho MATSUMOTO \& Masato WAKAYAMA

Alpha-determinant cyclic modules and Jacobi polynomials

MI2008-10 Sangyeol LEE \& Hiroki MASUDA

Jarque-Bera Normality Test for the Driving Lévy Process of a Discretely Observed Univariate SDE

MI2008-11 Hiroyuki CHIHARA \& Eiji ONODERA

A third order dispersive flow for closed curves into almost Hermitian manifolds

MI2008-12 Takehiko KINOSHITA, Kouji HASHIMOTO and Mitsuhiro T. NAKAO

On the $L^{2}$ a priori error estimates to the finite element solution of elliptic problems with singular adjoint operator

MI2008-13 Jacques FARAUT and Masato WAKAYAMA

Hermitian symmetric spaces of tube type and multivariate Meixner-Pollaczek polynomials 
MI2008-14 Takashi NAKAMURA

Riemann zeta-values, Euler polynomials and the best constant of Sobolev inequality

MI2008-15 Takashi NAKAMURA

Some topics related to Hurwitz-Lerch zeta functions

MI2009-1 Yasuhide FUKUMOTO

Global time evolution of viscous vortex rings

MI2009-2 Hidetoshi MATSUI \& Sadanori KONISHI

Regularized functional regression modeling for functional response and predictors

MI2009-3 Hidetoshi MATSUI \& Sadanori KONISHI

Variable selection for functional regression model via the $L_{1}$ regularization

MI2009-4 Shuichi KAWANO \& Sadanori KONISHI

Nonlinear logistic discrimination via regularized Gaussian basis expansions

MI2009-5 Toshiro HIRANOUCHI \& Yuichiro TAGUCHII

Flat modules and Groebner bases over truncated discrete valuation rings

MI2009-6 Kenji KAJIWARA \& Yasuhiro OHTA

Bilinearization and Casorati determinant solutions to non-autonomous $1+1$ dimensional discrete soliton equations

MI2009-7 Yoshiyuki KAGEI

Asymptotic behavior of solutions of the compressible Navier-Stokes equation around the plane Couette flow

MI2009-8 Shohei TATEISHI, Hidetoshi MATSUI \& Sadanori KONISHI

Nonlinear regression modeling via the lasso-type regularization

MI2009-9 Takeshi TAKAISHI \& Masato KIMURA

Phase field model for mode III crack growth in two dimensional elasticity

MI2009-10 Shingo SAITO

Generalisation of Mack's formula for claims reserving with arbitrary exponents for the variance assumption

MI2009-11 Kenji KAJIWARA, Masanobu KANEKO, Atsushi NOBE \& Teruhisa TSUDA Ultradiscretization of a solvable two-dimensional chaotic map associated with the Hesse cubic curve

MI2009-12 Tetsu MASUDA

Hypergeometric T -functions of the q-Painlevé system of type $E_{8}^{(1)}$

MI2009-13 Hidenao IWANE, Hitoshi YANAMI, Hirokazu ANAI \& Kazuhiro YOKOYAMA A Practical Implementation of a Symbolic-Numeric Cylindrical Algebraic Decomposition for Quantifier Elimination

MI2009-14 Yasunori MAEKAWA

On Gaussian decay estimates of solutions to some linear elliptic equations and its applications 
MI2009-15 Yuya ISHIHARA \& Yoshiyuki KAGEI

Large time behavior of the semigroup on $L^{p}$ spaces associated with the linearized compressible Navier-Stokes equation in a cylindrical domain

MI2009-16 Chikashi ARITA, Atsuo KUNIBA, Kazumitsu SAKAI \& Tsuyoshi SAWABE

Spectrum in multi-species asymmetric simple exclusion process on a ring

MI2009-17 Masato WAKAYAMA \& Keitaro YAMAMOTO

Non-linear algebraic differential equations satisfied by certain family of elliptic functions

MI2009-18 Me Me NAING \& Yasuhide FUKUMOTO

Local Instability of an Elliptical Flow Subjected to a Coriolis Force

MI2009-19 Mitsunori KAYANO \& Sadanori KONISHI

Sparse functional principal component analysis via regularized basis expansions and its application

MI2009-20 Shuichi KAWANO \& Sadanori KONISHI

Semi-supervised logistic discrimination via regularized Gaussian basis expansions

MI2009-21 Hiroshi YOSHIDA, Yoshihiro MIWA \& Masanobu KANEKO

Elliptic curves and Fibonacci numbers arising from Lindenmayer system with symbolic computations

MI2009-22 Eiji ONODERA

A remark on the global existence of a third order dispersive flow into locally Hermitian symmetric spaces

MI2009-23 Stjepan LUGOMER \& Yasuhide FUKUMOTO

Generation of ribbons, helicoids and complex scherk surface in laser-matter Interactions

MI2009-24 Yu KAWAKAMI

Recent progress in value distribution of the hyperbolic Gauss map

MI2009-25 Takehiko KINOSHITA \& Mitsuhiro T. NAKAO

On very accurate enclosure of the optimal constant in the a priori error estimates for $H_{0}^{2}$-projection

MI2009-26 Manabu YOSHIDA

Ramification of local fields and Fontaine's property (Pm)

MI2009-27 Yu KAWAKAMI

Value distribution of the hyperbolic Gauss maps for flat fronts in hyperbolic threespace

MI2009-28 Masahisa TABATA

Numerical simulation of fluid movement in an hourglass by an energy-stable finite element scheme

MI2009-29 Yoshiyuki KAGEI \& Yasunori MAEKAWA

Asymptotic behaviors of solutions to evolution equations in the presence of translation and scaling invariance 
MI2009-30 Yoshiyuki KAGEI \& Yasunori MAEKAWA

On asymptotic behaviors of solutions to parabolic systems modelling chemotaxis

MI2009-31 Masato WAKAYAMA \& Yoshinori YAMASAKI

Hecke's zeros and higher depth determinants

MI2009-32 Olivier PIRONNEAU \& Masahisa TABATA

Stability and convergence of a Galerkin-characteristics finite element scheme of lumped mass type

MI2009-33 Chikashi ARITA

Queueing process with excluded-volume effect

MI2009-34 Kenji KAJIWARA, Nobutaka NAKAZONO \& Teruhisa TSUDA

Projective reduction of the discrete Painlevé system of type $\left(A_{2}+A_{1}\right)^{(1)}$

MI2009-35 Yosuke MIZUYAMA, Takamasa SHINDE, Masahisa TABATA \& Daisuke TAGAMI Finite element computation for scattering problems of micro-hologram using DtN map

MI2009-36 Reiichiro KAWAI \& Hiroki MASUDA

Exact simulation of finite variation tempered stable Ornstein-Uhlenbeck processes

MI2009-37 Hiroki MASUDA

On statistical aspects in calibrating a geometric skewed stable asset price model

MI2010-1 Hiroki MASUDA

Approximate self-weighted LAD estimation of discretely observed ergodic OrnsteinUhlenbeck processes

MI2010-2 Reiichiro KAWAI \& Hiroki MASUDA

Infinite variation tempered stable Ornstein-Uhlenbeck processes with discrete observations

MI2010-3 Kei HIROSE, Shuichi KAWANO, Daisuke MIIKE \& Sadanori KONISHI

Hyper-parameter selection in Bayesian structural equation models

MI2010-4 Nobuyuki IKEDA \& Setsuo TANIGUCHI

The Itô-Nisio theorem, quadratic Wiener functionals, and 1-solitons

MI2010-5 Shohei TATEISHI \& Sadanori KONISHI

Nonlinear regression modeling and detecting change point via the relevance vector machine

MI2010-6 Shuichi KAWANO, Toshihiro MISUMI \& Sadanori KONISHI

Semi-supervised logistic discrimination via graph-based regularization

MI2010-7 Teruhisa TSUDA

UC hierarchy and monodromy preserving deformation

MI2010-8 Takahiro ITO

Abstract collision systems on groups 
MI2010-9 Hiroshi YOSHIDA, Kinji KIMURA, Naoki YOSHIDA, Junko TANAKA \& Yoshihiro MIWA

An algebraic approach to underdetermined experiments

MI2010-10 Kei HIROSE \& Sadanori KONISHI

Variable selection via the grouped weighted lasso for factor analysis models

MI2010-11 Katsusuke NABESHIMA \& Hiroshi YOSHIDA

Derivation of specific conditions with Comprehensive Groebner Systems

MI2010-12 Yoshiyuki KAGEI, Yu NAGAFUCHI \& Takeshi SUDOU

Decay estimates on solutions of the linearized compressible Navier-Stokes equation around a Poiseuille type flow

MI2010-13 Reiichiro KAWAI \& Hiroki MASUDA

On simulation of tempered stable random variates

MI2010-14 Yoshiyasu OZEKI

Non-existence of certain Galois representations with a uniform tame inertia weight

MI2010-15 Me Me NAING \& Yasuhide FUKUMOTO

Local Instability of a Rotating Flow Driven by Precession of Arbitrary Frequency

MI2010-16 Yu KAWAKAMI \& Daisuke NAKAJO

The value distribution of the Gauss map of improper affine spheres

MI2010-17 Kazunori YASUTAKE

On the classification of rank 2 almost Fano bundles on projective space

MI2010-18 Toshimitsu TAKAESU

Scaling limits for the system of semi-relativistic particles coupled to a scalar bose field

MI2010-19 Reiichiro KAWAI \& Hiroki MASUDA

Local asymptotic normality for normal inverse Gaussian Lévy processes with highfrequency sampling

MI2010-20 Yasuhide FUKUMOTO, Makoto HIROTA \& Youichi MIE

Lagrangian approach to weakly nonlinear stability of an elliptical flow

MI2010-21 Hiroki MASUDA

Approximate quadratic estimating function for discretely observed Lévy driven SDEs with application to a noise normality test

MI2010-22 Toshimitsu TAKAESU

A Generalized Scaling Limit and its Application to the Semi-Relativistic Particles System Coupled to a Bose Field with Removing Ultraviolet Cutoffs

MI2010-23 Takahiro ITO, Mitsuhiko FUJIO, Shuichi INOKUCHI \& Yoshihiro MIZOGUCHI Composition, union and division of cellular automata on groups

MI2010-24 Toshimitsu TAKAESU

A Hardy's Uncertainty Principle Lemma in Weak Commutation Relations of HeisenbergLie Algebra 
MI2010-25 Toshimitsu TAKAESU

On the Essential Self-Adjointness of Anti-Commutative Operators

MI2010-26 Reiichiro KAWAI \& Hiroki MASUDA

On the local asymptotic behavior of the likelihood function for Meixner Lévy processes under high-frequency sampling

MI2010-27 Chikashi ARITA \& Daichi YANAGISAWA

Exclusive Queueing Process with Discrete Time

MI2010-28 Jun-ichi INOGUCHI, Kenji KAJIWARA, Nozomu MATSUURA \& Yasuhiro OHTA Motion and Bäcklund transformations of discrete plane curves

MI2010-29 Takanori YASUDA, Masaya YASUDA, Takeshi SHIMOYAMA \& Jun KOGURE On the Number of the Pairing-friendly Curves

MI2010-30 Chikashi ARITA \& Kohei MOTEGI

Spin-spin correlation functions of the $q$-VBS state of an integer spin model

MI2010-31 Shohei TATEISHI \& Sadanori KONISHI

Nonlinear regression modeling and spike detection via Gaussian basis expansions

MI2010-32 Nobutaka NAKAZONO

Hypergeometric $\tau$ functions of the $q$-Painlevé systems of type $\left(A_{2}+A_{1}\right)^{(1)}$

MI2010-33 Yoshiyuki KAGEI

Global existence of solutions to the compressible Navier-Stokes equation around parallel flows

MI2010-34 Nobushige KUROKAWA, Masato WAKAYAMA \& Yoshinori YAMASAKI

Milnor-Selberg zeta functions and zeta regularizations

MI2010-35 Kissani PERERA \& Yoshihiro MIZOGUCHI

Laplacian energy of directed graphs and minimizing maximum outdegree algorithms

MI2010-36 Takanori YASUDA

CAP representations of inner forms of $S p(4)$ with respect to Klingen parabolic subgroup

MI2010-37 Chikashi ARITA \& Andreas SCHADSCHNEIDER

Dynamical analysis of the exclusive queueing process

MI2011-1 Yasuhide FUKUMOTO\& Alexander B. SAMOKHIN

Singular electromagnetic modes in an anisotropic medium

MI2011-2 Hiroki KONDO, Shingo SAITO \& Setsuo TANIGUCHI

Asymptotic tail dependence of the normal copula

MI2011-3 Takehiro HIROTSU, Hiroki KONDO, Shingo SAITO, Takuya SATO, Tatsushi TANAKA \& Setsuo TANIGUCHI

Anderson-Darling test and the Malliavin calculus

MI2011-4 Hiroshi INOUE, Shohei TATEISHI \& Sadanori KONISHI

Nonlinear regression modeling via Compressed Sensing 
MI2011-5 Hiroshi INOUE

Implications in Compressed Sensing and the Restricted Isometry Property

MI2011-6 Daeju KIM \& Sadanori KONISHI

Predictive information criterion for nonlinear regression model based on basis expansion methods

MI2011-7 Shohei TATEISHI, Chiaki KINJYO \& Sadanori KONISHI

Group variable selection via relevance vector machine

MI2011-8 Jan BREZINA \& Yoshiyuki KAGEI

Decay properties of solutions to the linearized compressible Navier-Stokes equation around time-periodic parallel flow

Group variable selection via relevance vector machine

MI2011-9 Chikashi ARITA, Arvind AYYER, Kirone MALLICK \& Sylvain PROLHAC

Recursive structures in the multispecies TASEP

MI2011-10 Kazunori YASUTAKE

On projective space bundle with nef normalized tautological line bundle

MI2011-11 Hisashi ANDO, Mike HAY, Kenji KAJIWARA \& Tetsu MASUDA

An explicit formula for the discrete power function associated with circle patterns of Schramm type

MI2011-12 Yoshiyuki KAGEI

Asymptotic behavior of solutions to the compressible Navier-Stokes equation around a parallel flow

MI2011-13 Vladimír CHALUPECKÝ \& Adrian MUNTEAN

Semi-discrete finite difference multiscale scheme for a concrete corrosion model: approximation estimates and convergence

MI2011-14 Jun-ichi INOGUCHI, Kenji KAJIWARA, Nozomu MATSUURA \& Yasuhiro OHTA Explicit solutions to the semi-discrete modified KdV equation and motion of discrete plane curves

MI2011-15 Hiroshi INOUE

A generalization of restricted isometry property and applications to compressed sensing

MI2011-16 Yu KAWAKAMI

A ramification theorem for the ratio of canonical forms of flat surfaces in hyperbolic three-space

MI2011-17 Naoyuki KAMIYAMA

Matroid intersection with priority constraints

MI2012-1 Kazufumi KIMOTO \& Masato WAKAYAMA

Spectrum of non-commutative harmonic oscillators and residual modular forms

MI2012-2 Hiroki MASUDA

Mighty convergence of the Gaussian quasi-likelihood random fields for ergodic Levy driven SDE observed at high frequency 
MI2012-3 Hiroshi INOUE

A Weak RIP of theory of compressed sensing and LASSO

MI2012-4 Yasuhide FUKUMOTO \& Youich MIE

Hamiltonian bifurcation theory for a rotating flow subject to elliptic straining field

MI2012-5 Yu KAWAKAMI

On the maximal number of exceptional values of Gauss maps for various classes of surfaces

MI2012-6 Marcio GAMEIRO, Yasuaki HIRAOKA, Shunsuke IZUMI, Miroslav KRAMAR, Konstantin MISCHAIKOW \& Vidit NANDA

Topological Measurement of Protein Compressibility via Persistence Diagrams

MI2012-7 Nobutaka NAKAZONO \& Seiji NISHIOKA

Solutions to a $q$-analog of Painlevé III equation of type $D_{7}^{(1)}$

MI2012-8 Naoyuki KAMIYAMA

A new approach to the Pareto stable matching problem

MI2012-9 Jan BREZINA \& Yoshiyuki KAGEI

Spectral properties of the linearized compressible Navier-Stokes equation around time-periodic parallel flow

MI2012-10 Jan BREZINA

Asymptotic behavior of solutions to the compressible Navier-Stokes equation around a time-periodic parallel flow

MI2012-11 Daeju KIM, Shuichi KAWANO \& Yoshiyuki NINOMIYA

Adaptive basis expansion via the extended fused lasso

MI2012-12 Masato WAKAYAMA

On simplicity of the lowest eigenvalue of non-commutative harmonic oscillators

MI2012-13 Masatoshi OKITA

On the convergence rates for the compressible

Navier- Stokes equations with potential force

MI2013-1 Abuduwaili PAERHATI \& Yasuhide FUKUMOTO

A Counter-example to Thomson-Tait-Chetayev's Theorem

MI2013-2 Yasuhide FUKUMOTO \& Hirofumi SAKUMA

A unified view of topological invariants of barotropic and baroclinic fluids and their application to formal stability analysis of three-dimensional ideal gas flows

MI2013-3 Hiroki MASUDA

Asymptotics for functionals of self-normalized residuals of discretely observed stochastic processes

MI2013-4 Naoyuki KAMIYAMA

On Counting Output Patterns of Logic Circuits

MI2013-5 Hiroshi INOUE

RIPless Theory for Compressed Sensing 
MI2013-6 Hiroshi INOUE

Improved bounds on Restricted isometry for compressed sensing

MI2013-7 Hidetoshi MATSUI

Variable and boundary selection for functional data via multiclass logistic regression modeling

MI2013-8 Hidetoshi MATSUI

Variable selection for varying coefficient models with the sparse regularization 